\title{
On the Existence of Polynomials with Chaotic Behaviour
}

\author{
Nilson C. Bernardes Jr. ${ }^{1}$ and Alfredo Peris ${ }^{2}$ \\ ${ }^{1}$ Departamento de Matemática Aplicada, Instituto de Matemática, Universidade Federal do Rio de Janeiro, CP 68530, \\ 21945-970 Rio de Janeiro, RJ, Brazil \\ ${ }^{2}$ IUMPA, Universitat Politècnica de València, Departament de Matemática Aplicada, Edifici 7A, 46022 València, Spain
}

Correspondence should be addressed to Alfredo Peris; aperis@mat.upv.es

Received 29 May 2013; Accepted 7 August 2013

Academic Editor: Ajda Fošner

Copyright (c) 2013 N. C. Bernardes Jr. and A. Peris. This is an open access article distributed under the Creative Commons Attribution License, which permits unrestricted use, distribution, and reproduction in any medium, provided the original work is properly cited.

We establish a general result on the existence of hypercyclic (resp., transitive, weakly mixing, mixing, frequently hypercyclic) polynomials on locally convex spaces. As a consequence we prove that every (real or complex) infinite-dimensional separable Frèchet space admits mixing (hence hypercyclic) polynomials of arbitrary positive degree. Moreover, every complex infinitedimensional separable Banach space with an unconditional Schauder decomposition and every complex Frèchet space with an unconditional basis support chaotic and frequently hypercyclic polynomials of arbitrary positive degree. We also study distributional chaos for polynomials and show that every infinite-dimensional separable Banach space supports polynomials of arbitrary positive degree that have a dense distributionally scrambled linear manifold.

\section{Introduction}

Let $X$ be a topological vector space over $\mathbb{K}=\mathbb{R}$ or $\mathbb{C}$. Given a continuous linear operator $T: X \rightarrow X$, recall that $T$ is said to be hypercyclic (resp., supercyclic) if there exists a vector $x \in X$ whose orbit $\left\{T^{n} x ; n \in \mathbb{N}\right\}$ (resp., whose projective orbit $\left\{\alpha T^{n} x ; n \in \mathbb{N}, \alpha \in \mathbb{K}\right\}$ ) is dense in $X$. In this case, such a vector $x$ is said to be hypercyclic (resp., supercyclic) for T. If, for every nonempty open set $U \subset X$, we have that

$$
\liminf _{N \rightarrow \infty} \frac{\left|\left\{n \leq N ; T^{n} x \in U\right\}\right|}{N}>0
$$

(where $|A|$ denotes the cardinality of $A \subset \mathbb{N}$ ), then $x$ is said to be a frequently hypercyclic vector for $T$, and the operator $T$ is called frequently hypercyclic. The operator $T$ is chaotic if it is hypercyclic and has a dense set of periodic points. The study of hypercyclic operators and, more generally, the area of linear dynamics has experienced a great development during the last 30 years. We refer the reader to the recent books $[1,2]$ for an up to date account of the subject.

A fundamental problem in this area was the following question on the existence of hypercyclic operators formulated by Rolewicz [3]:
Does every infinite-dimensional separable Banach space admit a hypercyclic operator?

Herzog [4] proved that the answer is "yes" if we replace the word "hypercyclic" by "supercyclic." Later, the question was finally answered affirmatively (and independently) by Ansari [5] and Bernal-González [6]. Bonet and Peris [7] extended their result to Fréchet spaces, so that we have the following existence theorem:

Every infinite-dimensional separable Fréchet space admits a hypercyclic operator.

The situation for chaos and frequent hypercyclicity is different: hereditarily indecomposable complex Banach spaces support neither chaotic operators [8] nor frequently hypercyclic operators [9]. The recent works $[10,11]$ show that, under very general conditions, we have a positive result:

Every complex infinite-dimensional separable Banach space with an unconditional Schauder decomposition and every complex Fréchet space with an unconditional basis support a chaotic and frequently hypercyclic operator. 
The study of hypercyclicity and supercyclicity for polynomials was initiated by Bernardes [12] who established the following results:

(i) no Banach space $X \neq\{0\}$ admits a hypercyclic homogeneous polynomial of degree $\geq 2$;

(ii) every infinite-dimensional separable Banach space admits supercyclic homogeneous polynomials of arbitrary positive degree.

Other results on the dynamics of homogeneous polynomials were obtained by Bernardes [13]. We refer the reader to the monograph [14] for polynomials on locally convex spaces. A map $Q: X \rightarrow X$ defined on a locally convex space $X$ is a homogeneous polynomial of degree $m$ (in short, $m$ homogeneous) if there is an $m$-linear continuous map $A$ : $X \times \cdots \times X \rightarrow X$ such that $Q x=A(x, \ldots, x)$ for any $x \in X$. A general polynomial of degree $n$ is a map $P: X \rightarrow X$ that can be written as $P=\sum_{m=0}^{n} Q_{m}$, where each $Q_{m}$ is an $m$-homogeneous polynomial on $X$ (by convenience, a 0 homogeneous polynomial is a constant map). In contrast to the case of Banach spaces, Peris [15] exhibited for each $m \geq 2$ an example of a chaotic homogeneous polynomial of degree $m$ on the product Fréchet space $\mathbb{C}^{\mathbb{N}}$. More examples of hypercyclic and chaotic homogeneous polynomials were obtained by Aron and Miralles [16] on certain spaces of continuous and differentiable functions. Also, Peris [17] obtained examples of chaotic nonhomogeneous polynomials on the Banach spaces $\ell_{p}(1 \leq p<\infty)$ and $c_{0}$. Moreover, Martínez-Giménez and Peris [18] found different types of chaotic homogeneous and nonhomogeneous polynomials on Köthe echelon spaces. A general result on the problem of existence of hypercyclic polynomials was obtained by Martínez-Giménez and Peris [19]:

Every complex infinite-dimensional separable Fréchet space admits hypercyclic polynomials of arbitrary positive degree.

However, the problem remained open for real scalars even in the case of Banach spaces.

In this paper, by using a different approach, we prove that every (real or complex) infinite-dimensional separable Fréchet space admits hypercyclic polynomials of arbitrary positive degree (Theorem 2). In fact, we derive this theorem from a more general result (Theorem 1), which can also be applied in situations where the underlying space is not a Fréchet space (Example 5). We also prove that every complex infinite-dimensional separable Banach space with an unconditional Schauder decomposition and every complex Fréchet space with an unconditional basis support chaotic and frequently hypercyclic polynomials of arbitrary positive degree (Theorem 3). Let us mention that there exists a real Banach space with an unconditional basis that does not admit a chaotic operator [10, Theorem 4.1]. Moreover, we also consider, for the first time, the existence of (weakly) mixing polynomials and the existence of polynomials that exhibit distributional chaos. In particular, we prove that every infinite-dimensional separable Banach space supports polynomials of arbitrary positive degree that are distributionally chaotic (Theorem 4), with a dense linear manifold as a scrambled set.

We should point out that in the linear case, there are many sufficient criteria for hypercyclic and chaotic properties (see, e.g., $[1,2,20,21]$ ), but no criterion is available for the hypercyclic or chaotic behaviour of polynomials. This fact shows the difficulties to obtain hypercyclic or chaotic polynomials.

\section{Existence of Hypercyclic, Chaotic, and Frequently Hypercyclic Polynomials}

If $T$ is a continuous map from a topological space $X$ into itself, recall that $T$ is said to be transitive (resp., mixing) if, for any pair $U, V$ of nonempty open subsets of $X$, there exists $n \geq 0$ (resp., $n_{0} \geq 0$ ) such that

$$
\left.T^{n}(U) \cap V \neq \emptyset \quad \text { (resp., } \forall n \geq n_{0}\right) .
$$

Moreover, $T$ is said to be weakly mixing if $T \times T$ is topologically transitive on $X \times X$. Birkhoff transitivity theorem [2, Theorem 1.16] asserts that if $X$ is separable, completely metrizable, and has no isolated point, then $T$ is transitive if and only if $T$ has a dense orbit.

Let us now establish our main result.

Theorem 1. Let $X$ be a locally convex space over $\mathbb{K}$. If $X$ admits a hypercyclic (resp., transitive, weakly mixing, mixing, frequently hypercyclic) operator that has an eigenvalue of modulus less than or equal to 1 , then $X$ admits hypercyclic (resp., transitive, weakly mixing, mixing, frequently hypercyclic) polynomials of arbitrary positive degree.

Proof. Suppose that $T$ is a hypercyclic (resp., transitive, weakly mixing, mixing, frequently hypercyclic) operator on $X$ that has an eigenvalue $\lambda$ with $|\lambda| \leq 1$. Let $u \in X \backslash\{0\}$ be an eigenvector of $T$ associated with the eigenvalue $\lambda$. Let $Y:=$ $\operatorname{span}\{u\}$, choose a vector $v \in X \backslash Y$, and put $Z:=\operatorname{span}\{u, v\}$. Fix an integer $m \geq 2$ and consider the $m$-homogeneous polynomial $Q: Z \rightarrow X$ given by

$$
Q(\alpha u+\beta v):=\beta^{m} u
$$

It follows from the Hahn-Banach Theorem that there exists a continuous linear projection $F$ from $X$ onto $Z$. Define

$$
P:=Q \circ F \text {. }
$$

Then, $P: X \rightarrow X$ is a nonzero continuous $m$-homogeneous polynomial such that

$$
P(x+y)=P x \quad \text { whenever } x \in X, y \in Y .
$$

Moreover,

$$
T \circ P=\lambda P .
$$

Indeed, if $x \in X$ and $F x=\alpha u+\beta v$, then

$$
\begin{aligned}
T(P x) & =T(Q(\alpha u+\beta v))=T\left(\beta^{m} u\right) \\
& =\beta^{m} T u=\lambda \beta^{m} u=\lambda P x,
\end{aligned}
$$

where we used (3) and the fact that $T u=\lambda u$. 
We claim that

$$
(I-P) \circ(I+P)=I=(I+P) \circ(I-P),
$$

which shows that the map $I+P$ is a homeomorphism from $X$ onto $X$. In fact, for every $x \in X$,

$$
\begin{aligned}
(I-P)((I+P)(x)) & =(I-P)(x+P x) \\
& =x+P x-P(x+P x)=x,
\end{aligned}
$$

because of (5). The second equality in (8) is analogous.

Now, we will prove by induction that

$$
(T+P \circ T-\lambda P)^{n}=(I+P) \circ T^{n}-\lambda^{n} P \quad \forall n \in \mathbb{N} .
$$

Since for $n=1$ we obviously have $T+P \circ T-\lambda P=(I+P)$ 。 $T-\lambda P$, let us assume that (10) holds for a certain $n \in \mathbb{N}$, and let us consider the case $n+1$. Fix $x \in X$. By using (5), (6), and the induction hypothesis, we obtain

$$
\begin{aligned}
(T+ & P \circ T-\lambda P)^{n+1}(x) \\
= & \left((I+P) \circ T^{n}-\lambda^{n} P\right)((T+P \circ T-\lambda P)(x)) \\
= & (I+P)\left(T^{n+1} x+T^{n}(P(T x))-\lambda T^{n}(P x)\right) \\
& -\lambda^{n} P(T x+P(T x)-\lambda P x) \\
= & (I+P)\left(T^{n+1} x+\lambda^{n} P(T x)-\lambda^{n+1} P x\right)-\lambda^{n} P(T x) \\
= & T^{n+1} x+\lambda^{n} P(T x)-\lambda^{n+1} P x+P\left(T^{n+1} x\right)-\lambda^{n} P(T x) \\
= & (I+P)\left(T^{n+1} x\right)-\lambda^{n+1} P x,
\end{aligned}
$$

as was to be shown.

Suppose that $P \circ T=\lambda P$. Then $P \circ T^{n}=\lambda^{n} P$ for all $n \geq$ 0 . Let $W$ and $U$ be disjoint open neighborhoods of 0 and $u$, respectively, with $W$ balanced. Since $T$ is transitive, there are $n \geq 0$ and $y \in P^{-1}(W)$ such that $T^{n} y \in P^{-1}(U)$. Hence,

$$
P\left(T^{n} y\right) \in U, \quad P\left(T^{n} y\right)=\lambda^{n} P y \in W,
$$

a contradiction. Thus, $P \circ T \neq \lambda P$, which proves that

$$
R:=T+P \circ T-\lambda P \text { is a polynomial of degree } m \text {. }
$$

By (10),

$$
R^{n}=(I+P) \circ T^{n}-\lambda^{n} P \quad \forall n \in \mathbb{N} .
$$

If $|\lambda|=1$ then the operator $\lambda^{-1} T$ is also hypercyclic (resp., transitive, weakly mixing, mixing, frequently hypercyclic) by [22] (resp., [23] and [2, Proposition 12.15], [24, Remark 5], and [1, Theorems 6.28 and 6.30]). Since $u$ is a fixed point of $\lambda^{-1} T$, by replacing the operator $T$ by the operator $\lambda^{-1} T$, we may assume that $\lambda=1$. In the case $T$ is hypercyclic, it is clear from (14) that $R$ is also hypercyclic. Assume $a \in X$ is a frequently hypercyclic vector for $T$ and fix a nonempty open set $U \subset X$. Let $V \subset X$ be a nonempty open set such that $(I+P)(V) \subset U+P a$. We have that

$$
\left\{n \in \mathbb{N} ; T^{n} a \in V\right\} \subset\left\{n \in \mathbb{N} ; R^{n} a \in U\right\},
$$

so that

$$
\liminf _{N \rightarrow \infty} \frac{\left|\left\{n \leq N ; R^{n} a \in U\right\}\right|}{N}>0,
$$

and $a$ is a frequently hypercyclic vector for $R$. Now, consider $T$ mixing. Let $U, V \subset X$ be nonempty open sets. Fix points $y \in U$ and $z \in V$, and put $w:=z+P y$. Since $w-P y=z \in V$, there are open neighborhoods $U^{\prime}$ of $y$ and $V^{\prime}$ of $w$ such that

$$
U^{\prime} \subset U, \quad V^{\prime}-P\left(U^{\prime}\right) \subset V .
$$

Let $n \in \mathbb{N}$ be such that $T^{n}\left(U^{\prime}\right) \cap(I+P)^{-1}\left(V^{\prime}\right) \neq \emptyset$. Fix $x \in U^{\prime}$ such that $T^{n} x \in(I+P)^{-1}\left(V^{\prime}\right)$. Then,

$$
x \in U, \quad R^{n} x=(I+P)\left(T^{n} x\right)-P x \in V^{\prime}-P\left(U^{\prime}\right) \subset V,
$$

proving that $R^{n}(U) \cap V \neq \emptyset$. This shows that $R$ is mixing. A similar reasoning shows that $R$ is weakly mixing (transitive) whenever $T$ is weakly mixing (transitive).

If $|\lambda|<1$ then $\lambda^{n} P \rightarrow 0$ pointwise on $X$. Hence, (14) implies that $R$ is hypercyclic if $T$ is hypercyclic, and $R$ is frequently hypercyclic if $T$ is frequently hypercyclic. Consider $T$ mixing and let $U, V \subset X$ be nonempty open sets. There are a nonempty open set $V^{\prime}$ and an open neighborhood $W$ of 0 such that

$$
V^{\prime}+W \subset V
$$

Moreover, there are a constant $C>0$ and a nonempty open set $U^{\prime}$ such that

$$
U^{\prime} \subset U, \quad P\left(U^{\prime}\right) \subset\{\alpha u ;|\alpha|<C\},
$$

and there exists $\varepsilon>0$ such that

$$
\{\alpha u ;|\alpha|<\varepsilon\} \subset W .
$$

Let $n \in \mathbb{N}$ be such that $|\lambda|^{n} C<\varepsilon$ and $T^{n}\left(U^{\prime}\right) \cap(I+$ $P)^{-1}\left(V^{\prime}\right) \neq \emptyset$. Fix $x \in U^{\prime}$ with $T^{n} x \in(I+P)^{-1}\left(V^{\prime}\right)$. Then,

$$
x \in U, \quad R^{n} x=(I+P)\left(T^{n} x\right)-\lambda^{n} P x \in V^{\prime}+W \subset V .
$$

This shows that $R$ is mixing. Similarly, $R$ is weakly mixing (transitive) if $T$ is weakly mixing (transitive).

Since every infinite-dimensional separable Fréchet space admits a mixing operator with a nonzero fixed point $[2$, Theorem 8.13], the previous theorem immediately implies the following result.

Theorem 2. Every infinite-dimensional separable Fréchet space over $\mathbb{K}$ admits mixing (hence hypercyclic) polynomials of arbitrary positive degree. 
Also, the existence of chaotic and frequently hypercyclic polynomials of arbitrary degree can be ensured under very general assumptions by the results in $[10,11]$.

Theorem 3. Every complex infinite-dimensional separable Banach space with an unconditional Schauder decompositionand every complex Fréchet space with an unconditional basis support chaotic and frequently hypercyclic polynomials of arbitrary positive degree.

Proof. Let $X$ satisfy the hypothesis. By $[10,11]$ there exists a chaotic and frequently hypercyclic operator $T: X \rightarrow X$ with a nonzero fixed point. Thus, with $\lambda=1$ in Theorem 1 , and for every integer $m \geq 2$, we know that there exists a frequently hypercyclic polynomial $R=T+P \circ T-P$ of degree $m$ on $X$ such that $R^{n}=(I+P) \circ T^{n}-P$ for every $n \in \mathbb{N}$. Since every periodic point of $T$ is also periodic for $R$, we immediately get that $R$ is also chaotic.

\section{Distributional Chaos for Polynomials on Banach Spaces}

The original notion of chaos in the mathematical literature comes from Li and Yorke's paper [25] on the study of dynamics of interval maps, and it concentrates on local aspects of dynamics of pairs. Schweizer and Smital introduced the concept of distributional chaos in [26] as a natural extension of the notion of chaos given by Li and Yorke.

There are several recent studies of distributional chaos for linear operators on Banach or Fréchet spaces (see, e.g., [21, 27-29]).

We recall that, given $A \subset \mathbb{N}$, its upper and lower densities are defined by

$$
\begin{aligned}
& \overline{\operatorname{dens}}(A):=\limsup _{n \rightarrow \infty} \frac{\operatorname{card}(A \cap[1, n])}{n}, \\
& \underline{\operatorname{dens}}(A):=\liminf _{n \rightarrow \infty} \frac{\operatorname{card}(A \cap[1, n])}{n},
\end{aligned}
$$

respectively. A continuous map $f: X \rightarrow X$ on a metric space $X$ is distributionally chaotic if there exist an uncountable set $\Gamma \subset X$ and $\varepsilon>0$ such that for every $\tau>0$ and each pair of distinct points $x, y \in \Gamma$, we have that

$$
\begin{aligned}
& \underline{\operatorname{dens}}\left\{n \in \mathbb{N}: d\left(f^{n}(x), f^{n}(y)\right)<\varepsilon\right\}=0, \\
& \overline{\text { dens }}\left\{n \in \mathbb{N}: d\left(f^{n}(x), f^{n}(y)\right)<\tau\right\}=1 .
\end{aligned}
$$

In this case, the set $\Gamma$ is a distributionally $\varepsilon$-scrambled set and the pair $(x, y)$ a distributionally chaotic pair. We say that $f$ is densely distributionally chaotic if the set $\Gamma$ may be chosen to be dense in $X$.

Inspired by the notion of irregular vector introduced by Beauzamy [30] for operators on Banach spaces, in [27] the authors defined the following notion: given an operator $T \in$ $B(X)$ on a Banach space $X$ and $x \in X, x$ is a distributionally irregular vector for $T$ if there are $A, B \subset \mathbb{N}$ with $\overline{\operatorname{dens}}(A)=$ $\overline{\operatorname{dens}}(B)=1$ such that

$$
\lim _{n \in A} T^{n} x=0, \quad \lim _{n \in B}\left\|T^{n} x\right\|=\infty .
$$

In this case we have, by linearity, that the set $\Gamma:=\operatorname{span}\{x\}$ is a distributionally $\varepsilon$-scrambled set, for every $\varepsilon>0$. If $M \subset$ $X$ is a linear manifold such that every nonzero $x \in M$ is a distributionally irregular vector for $T$, then $M$ is said to be a distributionally irregular manifold for $T$. By linearity, we also have that $\Gamma:=M$ is a distributionally $\varepsilon$-scrambled set, for every $\varepsilon>0$, if $M$ is a distributionally irregular manifold.

Theorem 4. If $X$ is an infinite-dimensional separable Banach space and $m \in \mathbb{N}, m>1$, then there is a distributionally chaotic polynomial of degree $m$ defined on $X$ with a dense distributionally scrambled linear manifold.

Proof. Let $u \in X$ be a nonzero vector and let $Y \subset X$ be a closed subspace complement of $\operatorname{span}\{u\}$. By Theorems 24 and 35 in [27], there exist an operator $\widetilde{T}: Y \rightarrow Y$, a dense subset $\left\{y_{m} ; m \in \mathbb{N}\right\} \subset Y$, a bounded sequence of linearly independent distributionally irregular vectors $\left(u_{m}\right)_{m}$ in $Y$, and a set of integers $A \subset \mathbb{N}$ with $\overline{\operatorname{dens}}(A)=1$ such that $\lim _{n} \widetilde{T}^{n}\left(y_{m}\right)=0$ and $\lim _{n \in A} \widetilde{T}^{n}\left(u_{m}\right)=0$ for each $m \in \mathbb{N}$. Let $\left\{r_{n} ; n \in \mathbb{N}\right\} \subset \mathbb{K}$ be a dense sequence in the scalar field and define the following countable set of vectors:

$$
z_{n, m}:=r_{n} u+y_{m}+\frac{1}{n m} u_{\phi(n, m)} \quad(n, m \in \mathbb{N}),
$$

where $\phi$ is a bijection from $\mathbb{N} \times \mathbb{N}$ onto $\mathbb{N}$. Extend $\widetilde{T}$ to an operator $T: X \rightarrow X$ by setting $T u=0$. By following the proof of [27, Theorem 24], we have that each nonzero $z \in M:=\operatorname{span}\left\{z_{n, m} ; n, m \in \mathbb{N}\right\}$ is a distributionally irregular vector for $T$. Moreover, $\lim _{n \in A} T^{n} z=0$ for every $z \in M$.

We construct the polynomial $P$ of degree $m$ as in Theorem 1, and we have that

$$
(T+P \circ T)^{n}=(I+P) \circ T^{n}
$$

for all $n \in \mathbb{N}$. The polynomial $R:=T+P \circ T$ has degree $m$ if we require $v=\widetilde{T} y$ for some nonzero $y \in Y$. Let now $z_{1}, z_{2} \in M$ with $z_{1} \neq z_{2}$. Since $\lim _{n \in A} T^{n} z_{1}=\lim _{n \in A} T^{n} z_{2}=0$, we have

$$
\begin{aligned}
\lim _{n \in A} & \left\|R^{n} z_{1}-R^{n} z_{2}\right\| \\
& =\lim _{n \in A}\left\|(I+P)\left(T^{n} z_{1}\right)-(I+P)\left(T^{n} z_{2}\right)\right\|=0 .
\end{aligned}
$$

Let $G: X \rightarrow Y$ be the projection onto $Y$ with kernel $\operatorname{span}\{u\}$. Let $C>0$ be such that $\|x\| \geq C\|G x\|$ for every $x \in X$. By the definition of $M, y:=G z_{1}-G z_{2} \neq 0$ is a distributionally irregular vector for $\widetilde{T}$. Then there is $B \subset \mathbb{N}$ with $\overline{\operatorname{dens}}(B)=1$ such that $\lim _{n \in B}\left\|\widetilde{T}^{n} y\right\|=\infty$. As a consequence,

$$
\begin{aligned}
\lim _{n \in B}\left\|R^{n} z_{1}-R^{n} z_{2}\right\| & \geq C \lim _{n \in B}\left\|G\left(R^{n} z_{1}-R^{n} z_{2}\right)\right\| \\
& =C \lim _{n \in B}\left\|\widetilde{T}^{n} y\right\|=\infty,
\end{aligned}
$$

and we conclude that $M$ is a distributionally scrambled dense linear manifold for the polynomial $R$. 


\section{Hypercyclic Polynomials on Non-Fréchet Spaces}

In this section we will present the existence of hypercyclic polynomials of arbitrary positive degree on certain general locally convex spaces which are not Fréchet as an application of Theorem 1 .

\section{Example 5.}

(1) Every Hausdorff countable inductive limit of separable Banach spaces $E=\operatorname{ind}_{n} E_{n}$ such that one of the steps $E_{m}$ is dense in $E$ admits hypercyclic polynomials of arbitrary positive degree. Actually, in Proposition 4 of [7] the existence of a hypercyclic operator $T: E \rightarrow$ $E$ such that 1 is an eigenvalue of $T$ was shown, and so the result follows from Theorem 1 .

(2) Every metrizable countably dimensional locally convex space $E$ has hypercyclic polynomials of any positive degree. Again, this is a consequence of the fact that there is a hypercyclic operator $T$ on $E$ with fixed nonzero vectors, as was recently observed in [31].

(3) Any direct sum $E=\oplus_{n} E_{n}$ of separable Fréchet spaces such that $E_{n}$ is infinite dimensional for infinitely many $n$ necessarily supports hypercyclic polynomials of arbitrary positive degree. In [32] it was shown that $E=$ $\oplus_{n} \ell_{p}, 1 \leq p<\infty$, and $E=\oplus_{n} s=\mathscr{D}(\Omega)$, where $s$ is the space of rapidly decreasing functions and $\mathscr{D}(\Omega)$ is the space of test functions on an open set $\Omega \subset \mathbb{R}^{m}$, support a noninjective hypercyclic operator. Shkarin [33] generalized this result to the present context by showing the existence of hypercyclic operators of the form $T=I+S$ for $S$ noninjective. Therefore the hypotheses of Theorem 1 are satisfied, and we conclude the result.

\section{Acknowledgments}

The present work was done while the first author was visiting the Departament de Matemática Aplicada at Universitat Politècnica de València (Spain). The first author is very grateful for the hospitality. The first author was supported in part by CAPES: Bolsista, Project no. BEX 4012/11-9. The second author was supported in part by MEC and FEDER, Project MTM2010-14909, and by GVA, Projects PROMETEO/2008/101 and PROMETEOII/2013/013.

\section{References}

[1] F. Bayart and E. Matheron, Dynamics of linear operators, vol. 179 of Cambridge Tracts in Mathematics, Cambridge University Press, Cambridge, Uk, 2009.

[2] K.-G. Grosse-Erdmann and A. Peris Manguillot, Linear Chaos, Universitext, Springer, London, UK, 2011.

[3] S. Rolewicz, "On orbits of elements," Studia Mathematica, vol. 32, pp. 17-22, 1969 .

[4] G. Herzog, "On linear operators having supercyclic vectors," Studia Mathematica, vol. 103, no. 3, pp. 295-298, 1992.
[5] S. I. Ansari, "Existence of hypercyclic operators on topological vector spaces," Journal of Functional Analysis, vol. 148, no. 2, pp. 384-390, 1997.

[6] L. Bernal-González, "On hypercyclic operators on Banach spaces," Proceedings of the American Mathematical Society, vol. 127, no. 4, pp. 1003-1010, 1999.

[7] J. Bonet and A. Peris, "Hypercyclic operators on non-normable Fréchet spaces," Journal of Functional Analysis, vol. 159, no. 2, pp. 587-595, 1998.

[8] J. Bonet, F. Martínez-Giménez, and A. Peris, "A Banach space which admits no chaotic operator," The Bulletin of the London Mathematical Society, vol. 33, no. 2, pp. 196-198, 2001.

[9] S. Shkarin, "On the spectrum of frequently hypercyclic operators," Proceedings of the American Mathematical Society, vol. 137, no. 1, pp. 123-134, 2009.

[10] M. De la Rosa, L. Frerick, S. Grivaux, and A. Peris, "Frequent hypercyclicity, chaos, and unconditional Schauder decompositions," Israel Journal of Mathematics, vol. 190, pp. 389-399, 2012.

[11] M. De la Rosa, L. Frerick, S. Grivaux, and A. Peris, "Chaos on Fréchet spaces with unconditional basis," Preprint.

[12] N. C. Bernardes, Jr., "On orbits of polynomial maps in Banach spaces," Quaestiones Mathematicae, vol. 21, no. 3-4, pp. 311-318, 1998.

[13] N. C. Bernardes, Jr., "A theorem on iterations of polynomial maps in several variables," Proceedings of the American Mathematical Society, vol. 126, no. 10, pp. 3037-3044, 1998.

[14] S. Dineen, Complex Analysis on Infinite-Dimensional Spaces, Springer Monographs in Mathematics, Springer, London, UK, 1999.

[15] A. Peris, "Erratum to "chaotic polynomials on Frèchet spaces," Proceedings of the American Mathematical Society, vol. 129, no. 12, pp. 3759-3760, 2001.

[16] R. M. Aron and A. Miralles, "Chaotic polynomials in spaces of continuous and differentiable functions," Glasgow Mathematical Journal, vol. 50, no. 2, pp. 319-323, 2008.

[17] A. Peris, "Chaotic polynomials on Banach spaces," Journal of Mathematical Analysis and Applications, vol. 287, no. 2, pp. 487493, 2003.

[18] F. Martínez-Giménez and A. Peris, "Chaotic polynomials on sequence and function spaces," International Journal of Bifurcation and Chaos in Applied Sciences and Engineering, vol. 20, no. 9, pp. 2861-2867, 2010.

[19] F. Martínez-Giménez and A. Peris, "Existence of hypercyclic polynomials on complex Fréchet spaces," Topology and its Applications, vol. 156, no. 18, pp. 3007-3010, 2009.

[20] J. Bès and A. Peris, "Disjointness in hypercyclicity," Journal of Mathematical Analysis and Applications, vol. 336, no. 1, pp. 297315, 2007.

[21] N. C. Bernardes, A. Bonilla, V. Müller, and A. Peris, "Distributional chaos for linear operators," Journal of Functional Analysis, vol. 265, no. 9, pp. 2143-2163, 2013.

[22] F. León-Saavedra and V. Müller, "Rotations of hypercyclic and supercyclic operators," Integral Equations and Operator Theory, vol. 50, no. 3, pp. 385-391, 2004.

[23] A. Manoussos, "A Birkhoff type transitivity theorem for nonseparable completely metrizable spaces with applications to linear dynamics," The Journal of Operator Theory. In press.

[24] K.-G. Grosse-Erdmann and A. Peris, "Weakly mixing operators on topological vector spaces," Revista de la Real Academia de Ciencias Exactas, Físicas y Naturales A, vol. 104, no. 2, pp. 413426, 2010. 
[25] T. Y. Li and J. A. Yorke, "Period three implies chaos," The American Mathematical Monthly, vol. 82, no. 10, pp. 985-992, 1975.

[26] B. Schweizer and J. Smítal, "Measures of chaos and a spectral decomposition of dynamical systems on the interval," Transactions of the American Mathematical Society, vol. 344, no. 2, pp. 737-754, 1994.

[27] T. Bermúdez, A. Bonilla, F. Martínez-Giménez, and A. Peris, "Li-Yorke and distributionally chaotic operators," Journal of Mathematical Analysis and Applications, vol. 373, no. 1, pp. 8393, 2011.

[28] B. Hou, P. Cui, and Y. Cao, "Chaos for Cowen-Douglas operators," Proceedings of the American Mathematical Society, vol. 138, no. 3, pp. 929-936, 2010.

[29] F. Martínez-Giménez, P. Oprocha, and A. Peris, "Distributional chaos for backward shifts," Journal of Mathematical Analysis and Applications, vol. 351, no. 2, pp. 607-615, 2009.

[30] B. Beauzamy, Introduction to Operator Theory and Invariant Subspaces, vol. 42, North-Holland Publishing, Amsterdam, The Netherlands, 1988.

[31] A. Schenke and S. Shkarin, "Hypercyclic operators on countably dimensional spaces," Journal of Mathematical Analysis and Applications, vol. 401, no. 1, pp. 209-217, 2013.

[32] J. Bonet, L. Frerick, A. Peris, and J. Wengenroth, "Transitive and hypercyclic operators on locally convex spaces," The Bulletin of the London Mathematical Society, vol. 37, no. 2, pp. 254-264, 2005.

[33] S. Shkarin, "Hypercyclic operators on topological vector spaces," Journal of the London Mathematical Society, vol. 86, no. 1, pp. 195-213, 2012. 


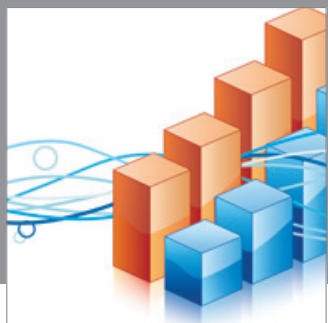

Advances in

Operations Research

mansans

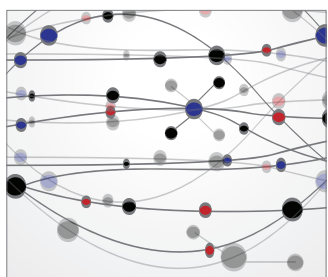

The Scientific World Journal
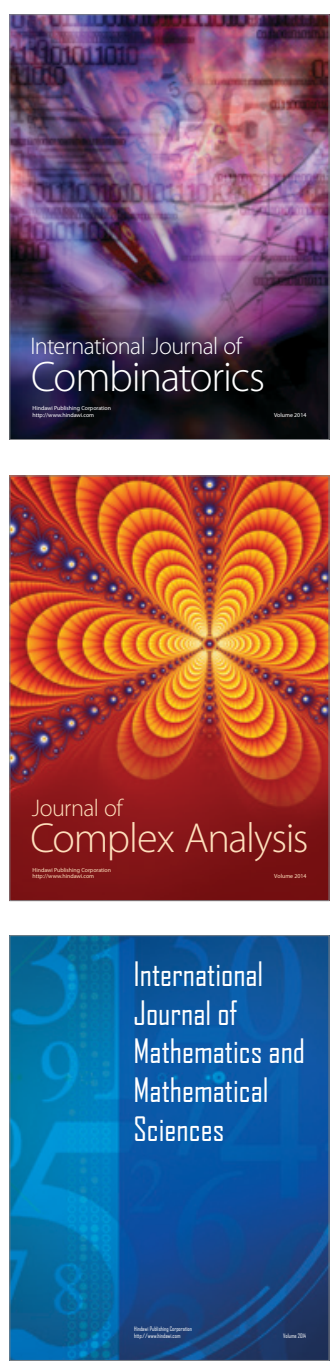
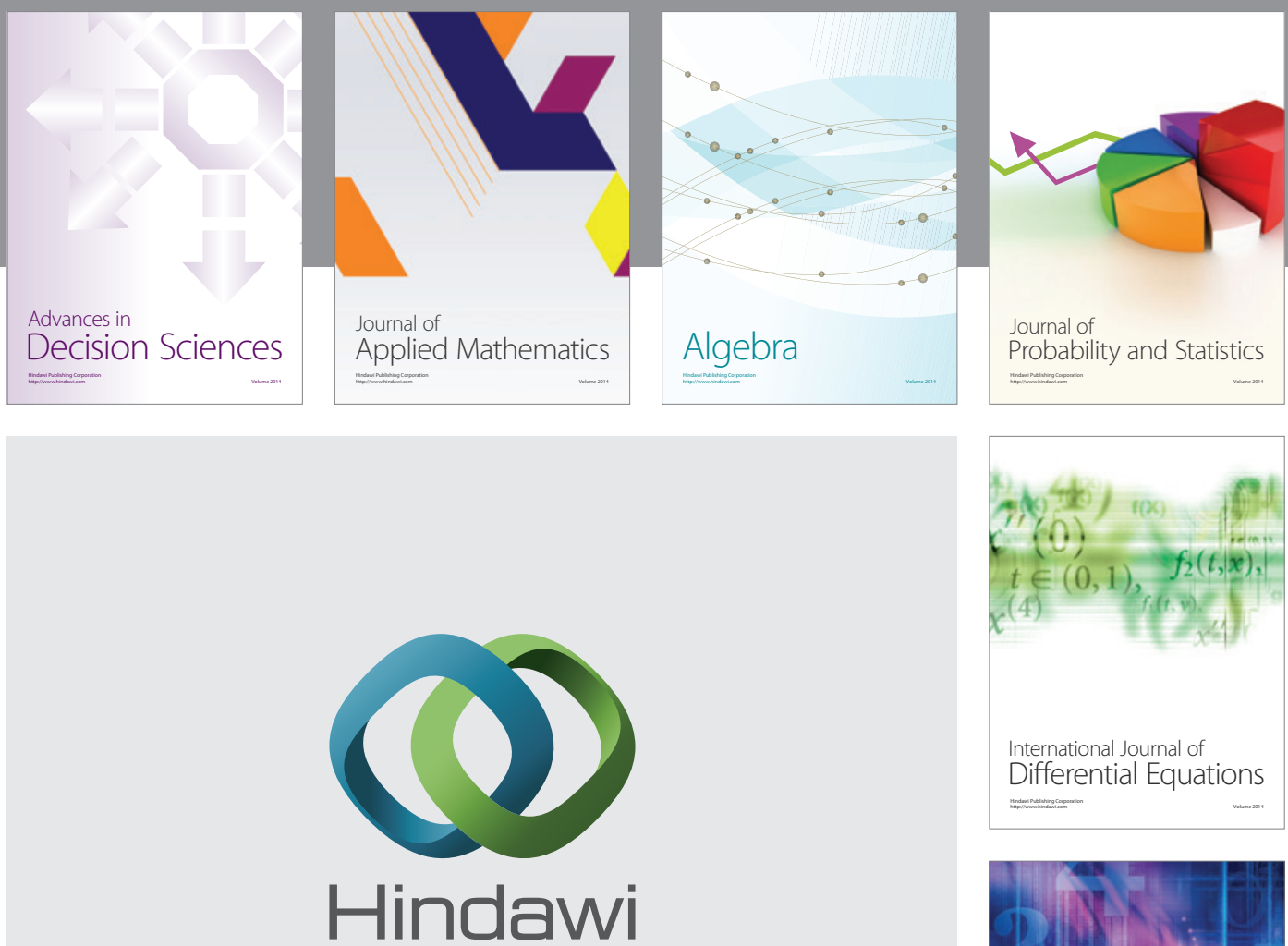

Submit your manuscripts at http://www.hindawi.com
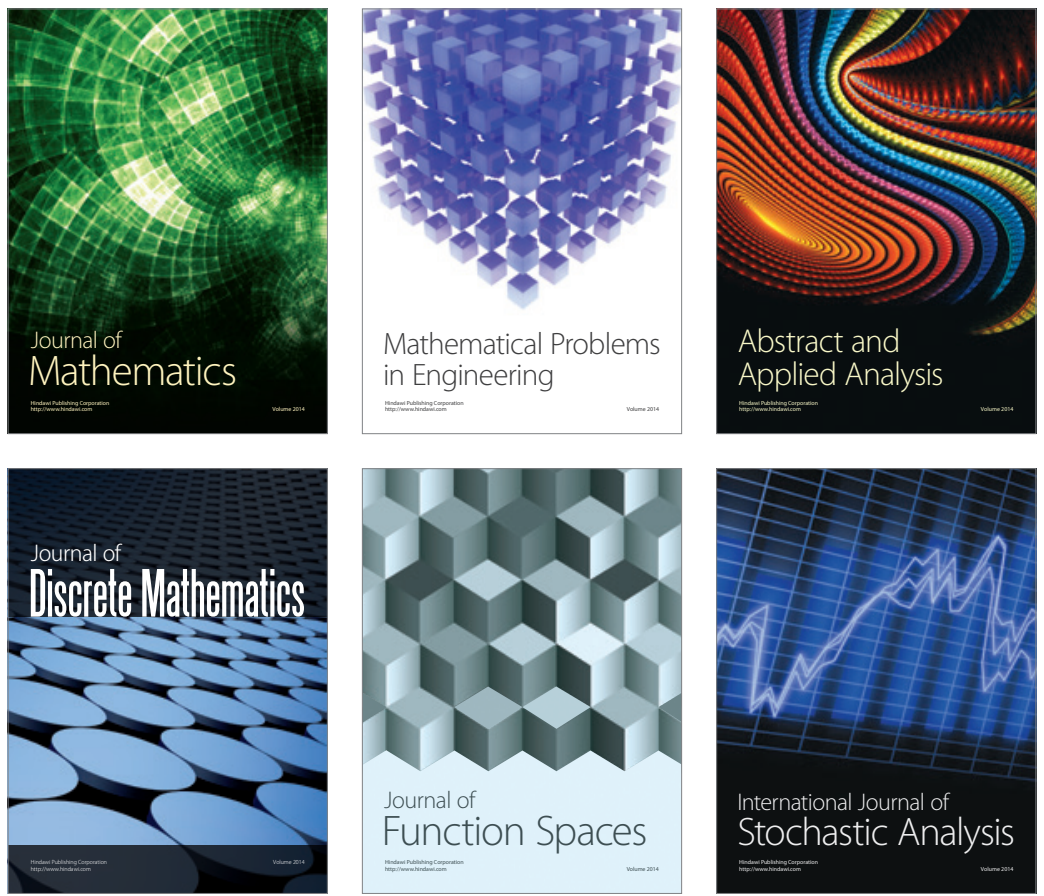

Journal of

Function Spaces

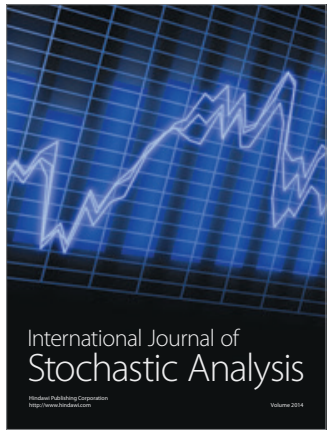

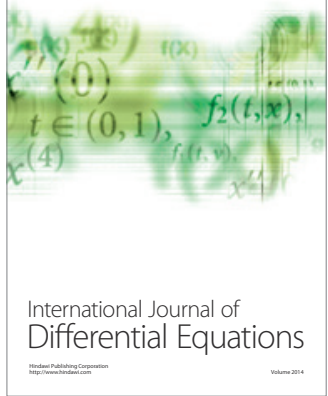
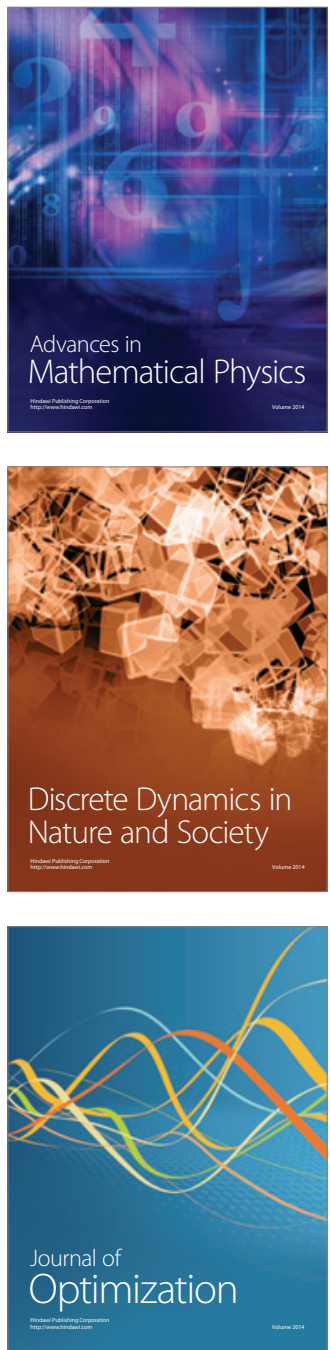